\title{
Encryption in Chaotic Systems with Sinusoidal Excitations
}

\author{
G. Obregón-Pulido, A. Torres-González, R. Cárdenas-Rodríguez, and G. Solís-Perales
}

División de Electrónica y Computación, Centro Universitario de Ciencias Exactas e Ingenierías (CUCEI), Universidad de Guadalajara, Avenida Revolución 1500, 44430 Guadalajara, JAL, Mexico

Correspondence should be addressed to G. Obregón-Pulido; obregonpulido@yahoo.com.mx

Received 4 December 2013; Revised 14 February 2014; Accepted 7 March 2014; Published 13 April 2014

Academic Editor: Hamid Reza Karimi

Copyright ( 92014 G. Obregón-Pulido et al. This is an open access article distributed under the Creative Commons Attribution License, which permits unrestricted use, distribution, and reproduction in any medium, provided the original work is properly cited.

\begin{abstract}
In this contribution an encryption method using a chaotic oscillator, excited by " $n$ " sinusoidal signals, is presented. The chaotic oscillator is excited by a sum of " $n$ " sinusoidal signals and a message. The objective is to encrypt such a message using the chaotic behavior and transmit it, and, as the chaotic system is perturbed by the sinusoidal signal, the transmission security could be increased due to the effect of such a perturbation. The procedure is based on the regulation theory and consider that the receiver knows the frequencies of the perturbing signal, with this considerations the algorithm estimates the excitation in such a way that the receiver can cancel out the perturbation and all the undesirable dynamics in order to produce only the message. In this way we consider that the security level is increased.
\end{abstract}

\section{Introduction}

Since Pecora and Carroll presented their work about chaos synchronization [1], the investigation on chaos has received significant attention and the phenomenon has spread to the application in communications security $([2-4])$. The use of chaotic systems for encoding and decoding is a new method different from the conventional techniques; see, for example, the work presented by Cuomo and Oppenheim in which a voice signal is encrypted by chaos and then is sent by the communication channel and is retrieved successfully [5].

In recent years, encryption schemes are being studied and increasing demand exists to develop a safer encryption system for transmitting data in real time via the Internet, wireless networks, and other devices ([6,7]).

The traditional standard encryption algorithm for images and data (DES) has a disadvantage when handling large amounts of data [8] and is performed digitally (first on a PC), and then sends the encrypted signal.

The online encryption in a dynamic system has the advantage of processing the signal in real time, so the analog signal (message) is encrypted while being sent.

In recent years some works were presented for the synchronization of master-slave structure ([9-11]), in the cites references a perturbation signal was introduced in the slave system and no perturbation was introduced in the master system. In different away, in the present work, a perturbation defined by the combination of the message and a sinusoidal signal is introduced in the master system.

In this work and algorithm is proposed in order to encrypt the message using a nonlinear chaotic system; this message is combined with " $n$ " sinusoidal signals whose amplitudes, phases, and bias are all unknown.

These signals excite the chaotic oscillator (the master) in order to encrypt the message more safely in the sense that the message signal is combined with sinusoidal signals; this combination increases the harmonics produced by the chaotic system and then the spectrum of the sending signal has more frequencies in the bandwidth of the signal.

In the proposed scheme the receiver (the slave) knows the frequencies to be used and estimates the sinusoidal signals which perturb the message and retrieve the message in exacta away.

In many other works (see [2-5]) only the message is used to excite the chaotic dynamical system and then is retrieved by the receptor. This is the principal difference in this work.

The paper is organized as follows: in Section 2 the problem is presented, the nonlinear estimator is designed in Section 3, in Section 4 several examples are shown, and finally in Section 5 some conclusions are presented. 


\section{Statement of the Problem}

In this paper a nonlinear system is proposed, which is excited by a signal given by the following equation:

$$
p(t)=B+\sum_{i=1}^{n} A_{i} \sin \left(\alpha_{i} t+\varphi_{i}\right)
$$

where the amplitudes $A_{i} \neq 0$, the phases $\varphi_{i}$, and the constant bias $B$ are unknown. In addition to the sinusoidal perturbation (1), an information signal " $m(t)$ " will be added in order to encrypt this message by the nonlinear dynamical chaotic system.

It is observed that the signal perturbation $p(t)$ can be generated by a linear system

$$
\dot{w}=S w
$$

which is called exosystem [12].

Various types of chaotic systems can be treated under the impulse of a sinusoidal input signal. In general the chaotic system to the encryption of the message can be seen in (3), where $f\left(x_{1}, x_{2}, \eta\right)$ and $\alpha\left(x_{1}, x_{2}\right)$ are nonlinear functions of the states, $p(t)$ is the sinusoidal perturbation, and $m(t)$ is the message to be encrypted:

$$
\begin{aligned}
& \dot{x}_{1}=x_{2}, \\
& \dot{x}_{2}=-a_{1} x_{1}-a_{2} x_{2}+f\left(x_{1}, x_{2}, \eta\right)+m(t)+p(t), \\
& \dot{\eta}=-a_{3} \eta+\alpha\left(x_{1}, x_{2}\right) .
\end{aligned}
$$

Having described the chaotic system model in general, we proceed to select the outputs of the system, which are given by

$$
\begin{aligned}
& y_{0}=x_{1}+x_{2}+f\left(x_{1}, x_{2}, \eta\right)+m(t), \\
& y_{1}=x_{1} .
\end{aligned}
$$

Some chaotic oscillators are studied in the following paragraphs and will be adapted to realize the desired encryption and take the form (3).

It is proposed that the outputs given by (4) can recover the message $m(t)$ and the disturbance signal $p(t)$ as shown in Figure 1.

If an intruder is capable of obtaining the transmission signal and can eliminate the chaos, find that the message will be disturbed by a sinusoidal signal and its harmonics as this was injected into the chaotic system, so the message is not decoded and the information will be preserved.

Note. Note that even though two outputs are taken, it is possible to transmit a single signal, if we perform a frequency multiplexing to send the two signals into one channel and they can be recovered later in the receiver.

In what follows some chaotic systems and how they are processed to take a proper structure are presented.

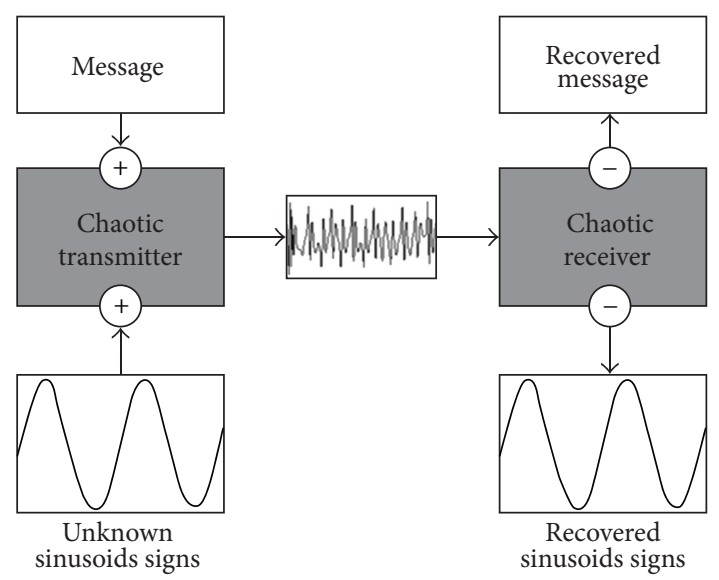

FIGURE 1: Estimation of disturbance $p(t)$ and message $m(t)$ in the chaotic system.

2.1. The Van der Pol Chaotic System. The Van der Pol equation provides an example of a nonlinear oscillator system. The system can be written as

$$
\ddot{x}-\mu\left(1-x^{2}\right) \dot{x}+x=p+m ;
$$

taking $x_{1}=x$ and $x_{2}=\dot{x}$, the system takes the form (2):

$$
\begin{aligned}
& \dot{x}_{1}=x_{2}, \\
& \dot{x}_{2}=-x_{1}+\mu x_{2}-\mu x_{1}^{2} x_{2}+p+m
\end{aligned}
$$

with the following coefficients:

$$
a_{1}=1, \quad a_{2}=-\mu, \quad f\left(x_{1}, x_{2}, \eta\right)=-\mu x_{1}^{2} x_{2} .
$$

2.2. The Duffing Chaotic System. Duffing equation is introduced in 1918 as a nonlinear oscillator model. The equation is defined as

$$
\ddot{x}+\delta \dot{x}-\beta x+x^{3}=p+m ;
$$

taking $x_{1}=x$ and $x_{2}=\dot{x}$, the system takes form (2):

$$
\begin{aligned}
& \dot{x}_{1}=x_{2}, \\
& \dot{x}_{2}=\beta x_{1}-\delta x_{2}-x_{1}^{3}+p+m
\end{aligned}
$$

with the following coefficients:

$$
a_{1}=-\beta, \quad a_{2}=\delta, \quad f\left(x_{1}, x_{2}, \eta\right)=-x_{1}^{3} .
$$

2.3. The Lorenz Chaotic System. This system is known as a simplified model of multiple physical systems $([13,14])$. This system is described by

$$
\begin{aligned}
& \dot{x}=a(y-x), \\
& \dot{y}=c x-y-x z+\frac{(m+p)}{a}, \\
& \dot{z}=-b z+x y .
\end{aligned}
$$


If we take $x_{1}=x, x_{2}=a(y-x)$, and $\eta=z$, the system (11) takes the form

$$
\begin{gathered}
\dot{x}_{1}=x_{2}, \\
\dot{x}_{2}=-a(1-c) x_{1}-(a+1) x_{2}-a x_{1} \eta+m+p \\
\dot{\eta}=-b \eta+x_{1}\left(\frac{x_{2}}{a}+x_{1}\right)
\end{gathered}
$$

and then takes the same structure as (2) where the respective coefficients are

$$
\begin{gathered}
a_{1}=a(1-c), \quad a_{2}=(a+1), \quad a_{3}=b, \\
f\left(x_{1}, x_{2}, \eta\right)=-a x_{1} \eta, \quad \alpha\left(x_{1}, x_{2}\right)=x_{1}\left(\frac{x_{2}}{a}+x_{1}\right) .
\end{gathered}
$$

Note. It is not difficult to see that chaotic systems of Lu, Chen, and Raylegh can be also put in form (2). Also in some cases the state $\eta$ does not exist ([11]).

For this class of systems some assumptions are considered.

Assumption 1. The constants $\left(a_{1}, a_{2}, a_{3}\right) \in \mathbb{R}$, the function $f\left(x_{1}, x_{2}, \eta\right)$, and $\eta$ dynamics are known.

Assumption 2. The $\alpha_{i}$ frequencies are known and $\alpha_{i} \neq \alpha_{j}$ for $i \neq j$.

Assumption 3. The message and the perturbation do not destroy the chaos.

With this assumption outputs (4) are chaotic and then this signal has an infinite number of frequencies.

\section{Receiver Design}

Now in order to recover the transmitted message, a state estimator for system (3) is proposed. In the estimator the information is decrypted and separated from the signal perturbation $p(t)$.

Consider the next theorem.

Theorem 4. Take the outputs signals given by (4) and consider Assumptions 1 to 3; then the estimator given by

$$
\begin{aligned}
& \dot{\hat{x}}_{1}=\widehat{x}_{2}+g_{1}\left(y_{1}-\widehat{y}_{1}\right), \\
& \dot{\hat{x}}_{2}=-a_{1} \widehat{x}_{1}-a_{2} \widehat{x}_{2}+\left(y_{0}-\widehat{y}_{0}\right)+g_{2}\left(y_{1}-\widehat{y}_{1}\right)+\widehat{p}, \\
& \dot{\hat{\eta}}=-a_{3} \widehat{\eta}+\alpha\left(\widehat{x}_{1}, \widehat{x}_{2}\right), \\
& \dot{\hat{w}}=S \widehat{w}+G_{0}\left(y_{1}-\widehat{y}_{1}\right),
\end{aligned}
$$

with

$$
\begin{aligned}
& \widehat{p}=\sum_{k=0}^{n} \widehat{w}_{2 k+1}, \quad S=\left[\begin{array}{ccccc}
S_{1} & 0 & \cdots & 0 & 0 \\
0 & S_{2} & \cdots & 0 & 0 \\
\vdots & \vdots & \ddots & \vdots & \vdots \\
0 & 0 & \cdots & S_{n} & 0 \\
0 & 0 & \cdots & 0 & 0
\end{array}\right], \\
& S_{j}=\left[\begin{array}{cc}
0 & \alpha_{j} \\
-\alpha_{j} & 0
\end{array}\right], \quad \widehat{m}=y_{0}-\widehat{y}_{0}-f\left(\widehat{x}_{1}, \widehat{x}_{2}, \widehat{\eta}\right)
\end{aligned}
$$

and outputs

$$
\begin{aligned}
& \widehat{y}_{0}=\widehat{x}_{1}+\widehat{x}_{2}, \\
& \widehat{y}_{1}=\widehat{x}_{1},
\end{aligned}
$$

where the constants $g_{1}, g_{2}$, and $G_{0}$ are properly selected, is such that the signals are estimated; it is

$$
\begin{gathered}
\widehat{x}_{1} \longrightarrow x_{1}, \quad \hat{x}_{2} \longrightarrow x_{2}, \quad \hat{\eta} \longrightarrow \eta, \\
\widehat{p} \longrightarrow p, \quad \widehat{m} \longrightarrow m .
\end{gathered}
$$

Proof. Consider the error system between (3) and (14) and the exosystem (2):

$$
\begin{aligned}
& \dot{e}_{1}=e_{2}-g_{1} e_{1}, \\
& \dot{e}_{2}=-\left(a_{1}+1\right) e_{1}-\left(a_{2}+1\right) e_{2}+\sum_{k=0}^{n} e_{w_{2 k+1}}-g_{2} e_{1}, \\
& \dot{e}_{3}=-a_{3} e_{3}+\alpha\left(x_{1}, x_{2}\right)-\alpha\left(\widehat{x}_{1}, \widehat{x}_{2}\right), \\
& \dot{e}_{w}=S e_{w}-G_{0} e_{1},
\end{aligned}
$$

where the errors are defined as

$$
\begin{aligned}
e_{1}=x_{1}-\widehat{x}_{1}, \quad e_{2} & =x_{2}-\widehat{x}_{2}, \quad e_{3}=\eta-\widehat{\eta}, \\
e_{w} & =w-\widehat{w} .
\end{aligned}
$$

From (16) consider the subsystem

$$
\begin{gathered}
{\left[\begin{array}{c}
\dot{e}_{1} \\
\dot{e}_{2} \\
\dot{e}_{w}
\end{array}\right]=\left[\begin{array}{ccc}
-g_{1} & 1 & 0 \\
-\left(a_{1}+1+g_{2}\right) & -\left(a_{2}+1\right) & V_{0} \\
-G_{0} & 0 & S
\end{array}\right]\left[\begin{array}{l}
e_{1} \\
e_{2} \\
e_{w}
\end{array}\right],} \\
V_{0}=\left[\begin{array}{llllll}
1 & 0 & 1 & \cdots & 0 & 1
\end{array}\right] .
\end{gathered}
$$

Then using control theory [15], it is possible to find constants $g_{1}, g_{2}$, and $G_{0}$ such that $e_{1} \rightarrow 0, e_{2} \rightarrow 0$, and $e_{w} \rightarrow 0$.

For the dynamics of $e_{3}$, it is observed that the difference between the nonlinear functions is an input to a stable linear system and it also tends to zero, so $e_{3}$ also tend to zero.

Then $\widehat{x}_{1} \rightarrow x_{1}, \widehat{x}_{2} \rightarrow x_{2}, \widehat{\eta} \rightarrow \eta$, and $\widehat{p} \rightarrow p$; for the message we can write

$$
\begin{aligned}
& \widehat{m}=y_{0}-\widehat{y}_{0}-f\left(\widehat{x}_{1}, \widehat{x}_{2}, \widehat{\eta}\right) \\
& \widehat{m}=x_{1}+x_{2}+f\left(x_{1}, x_{2}, \eta\right)+m-\left(\widehat{x}_{1}+\widehat{x}_{2}\right)-f\left(\widehat{x}_{1}, \widehat{x}_{2}, \widehat{\eta}\right) \\
& \widehat{m}=e_{1}+e_{2}+m+f\left(x_{1}, x_{2}, \eta\right)-f\left(\widehat{x}_{1}, \widehat{x}_{2}, \widehat{\eta}\right)
\end{aligned}
$$

and then $\widehat{m} \rightarrow m$, and the proof is finished. 


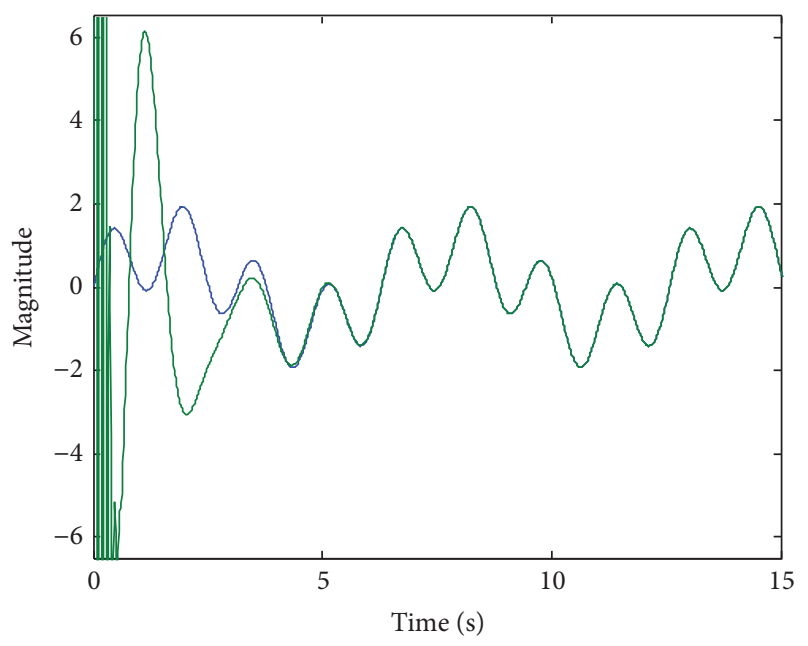

Figure 2: Estimation of disturbance on chaotic system. $(p(t)=$ $\sin (t)+\sin (4 t))$.

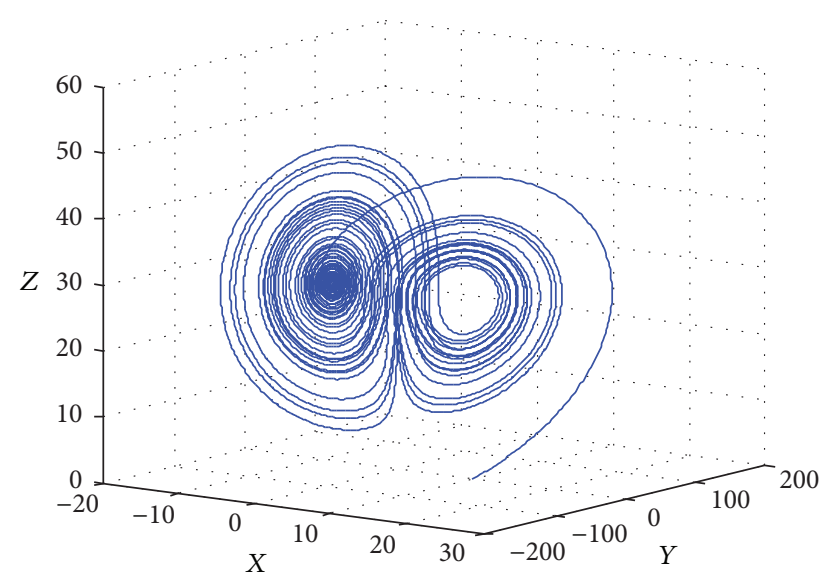

FIGURE 3: Lorenz attractor with sinusoidal signal and audio signal.

\section{Simulation}

4.1. Audio Signals. Consider the Lorenz oscillator given by equations

$$
\begin{aligned}
& \dot{x}=a(y-x), \\
& \dot{y}=c x-y-x z+\frac{(m+p)}{a}, \\
& \dot{z}=-b z+x y,
\end{aligned}
$$

where $m$ represents an audio signal from the song Un bel di vedremo (taken from the opera Madama Butterfly, Act II) and the disturbance signal with two frequencies is $p(t)=\sin (t)+$ $\sin (4 t)$.

Applying (14) with the following parameters $a=10, b=$ $28, c=8 / 3$ (these values generate chaotic behavior), and $g_{1}=$ $18 ; g_{2}=392 ; G_{0}=[1085,441,904,707]$ we can see how the disturbance $p(t)$ is estimated in Figure 2.

Figure 3 presents the chaotic behavior of the Lorenz attractor with the sinusoidal signal and audio signal.

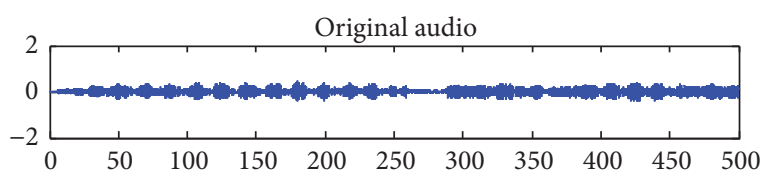

(a)

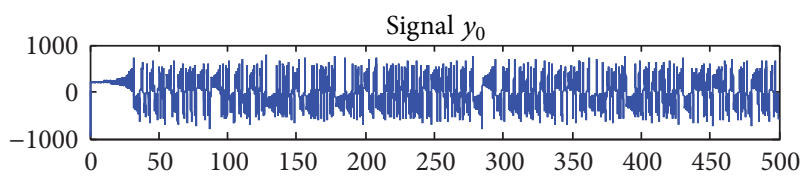

(b)

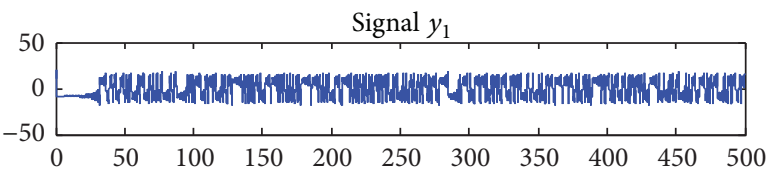

(c)

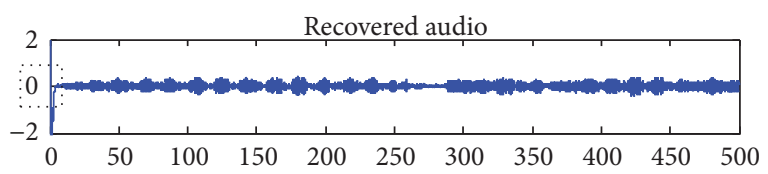

(d)

Figure 4: (a) the original audio signal to be encrypted. ((b), (c)) the outputs $y_{0}$ and $y_{1}$, of the encryption system, (d) the original audio signal recovered by the algorithm.

Figure 4 shows the original audio signal, chaotic signals $\left(y_{0}\right.$ and $\left.y_{1}\right)$, and audio recovery. The time axis is scaled. It is clear how the message is recovered in a very good manner.

In Figure 5, you can see how the recovered audio signal is the same as the original audio signal.

Figure 6 presents the error signal between the original audio signal and retrieved message signal. It is possible to see that the error tends to zero.

It is observed how the message and the perturbation are retrieved in exact manner, it is, the synchronization is exact and does not exist an error as other algorithms.

4.2. Digital Images. The method can also be applied to encrypt and to transmit digital images; for encryption, the image is modified to be sent in vector form. In this case, the receiver knows the number of rows and columns.

In this example we use the Duffing chaotic system with the perturbation given by $p(t)=\sin (0.5 t)$ :

$$
\begin{aligned}
& \dot{x}_{1}=x_{2}, \\
& \dot{x}_{2}=\beta x_{1}-\delta x_{2}-x_{1}^{3}+p+m,
\end{aligned}
$$

with the gain parameters being $g_{1}=20 ; g_{2}=100$; and $G_{0}=$ [350.7, 89.3516].

Figure 7 shows the sinusoidal signal estimated in comparison with the original signal.

Figure 8 represents the chaotic behavior of the Duffing attractor with sinusoidal signal and digital image (the reference image is Frida Kahlo). 


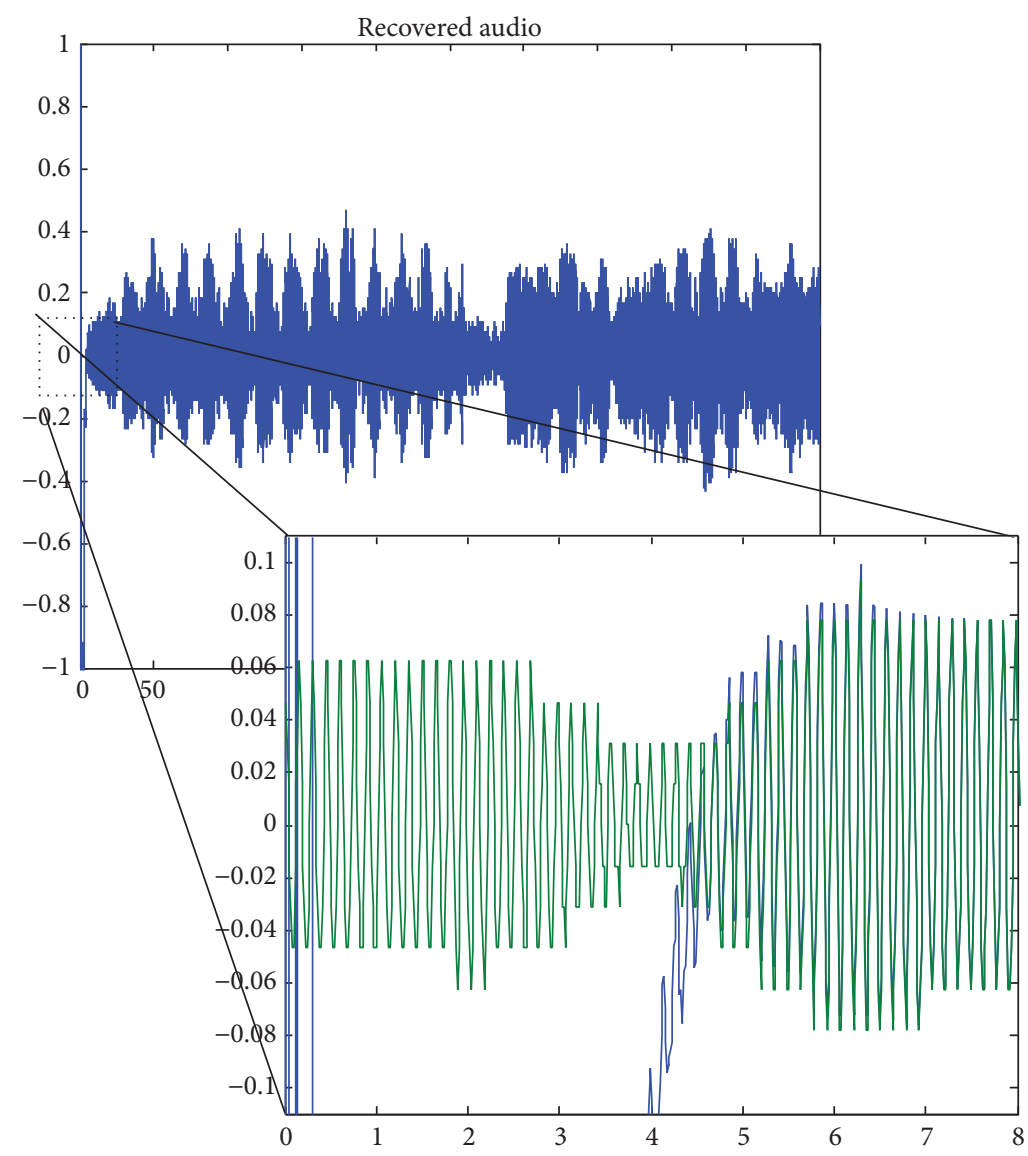

FIGURE 5: Original audio signal and recovered signal from the decoder implementation.

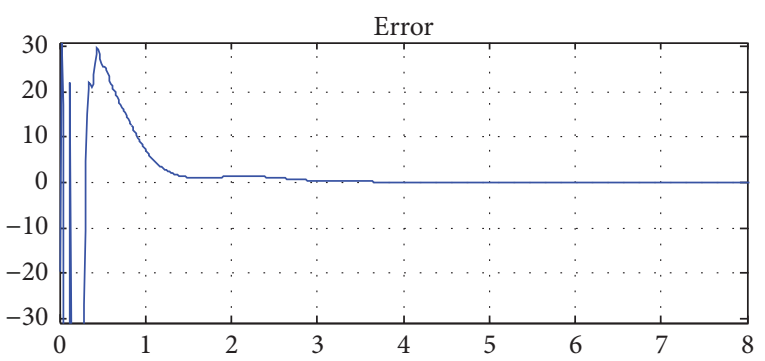

FIgURE 6: Error signal between the original message and the message retrieved.

Figure 9 represents the chaotic behavior of the Duffing attractor with sinusoidal signal and digital image (the reference image is Chichen-Itzá).

The differences between Figures 8 and 9 are due to the information of the images being different and therefore the perturbation changes the chaotic attractor in different manner; however, both figures show chaotic behavior.

Two examples are shown in Figures 10 and 11. First, image (a) is the original information and the recovered image. Second, image (b) is the chaotic signals $y_{0}$ and $y_{1}$.

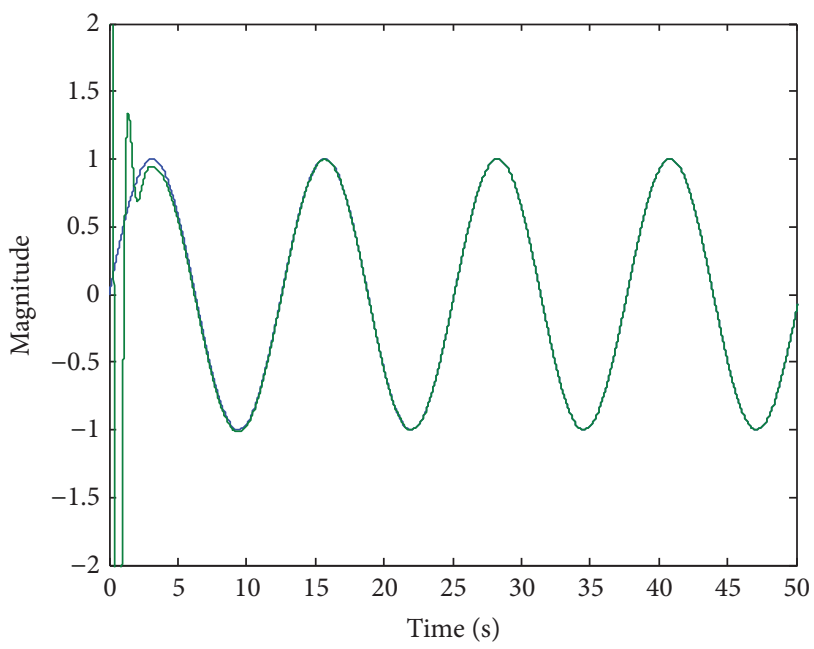

FIGURE 7: Sinusoidal signal retrieved and original signal.

\section{Conclusions}

In this contribution we presented a methodology to encrypt and transmit audio and image signals using chaos and regulation theory. The algorithm consisted in perturbing a chaotic system with a signal composed of $n$ sinusoidal signals with 


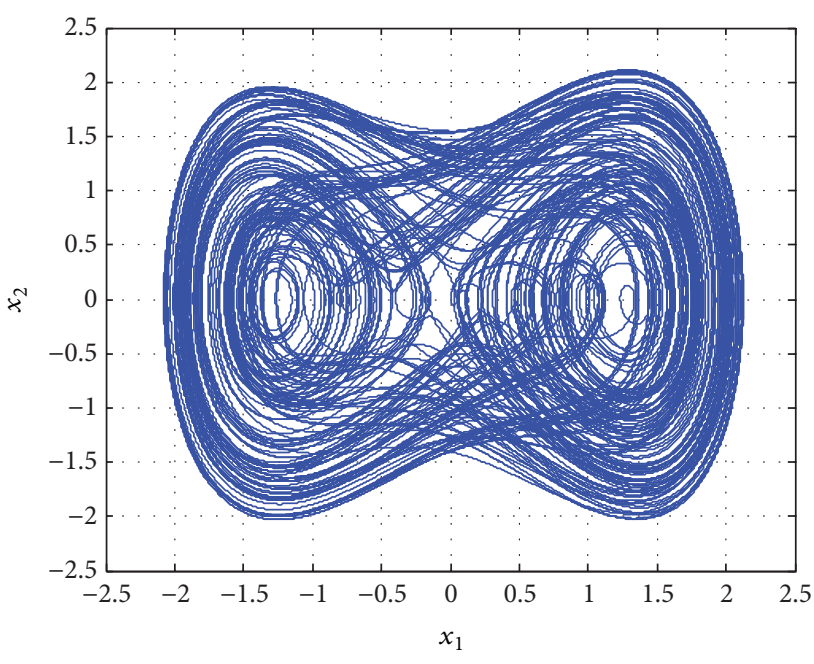

Figure 8: Duffing attractor with sinusoidal signal and digital image (Frida Kahlo).

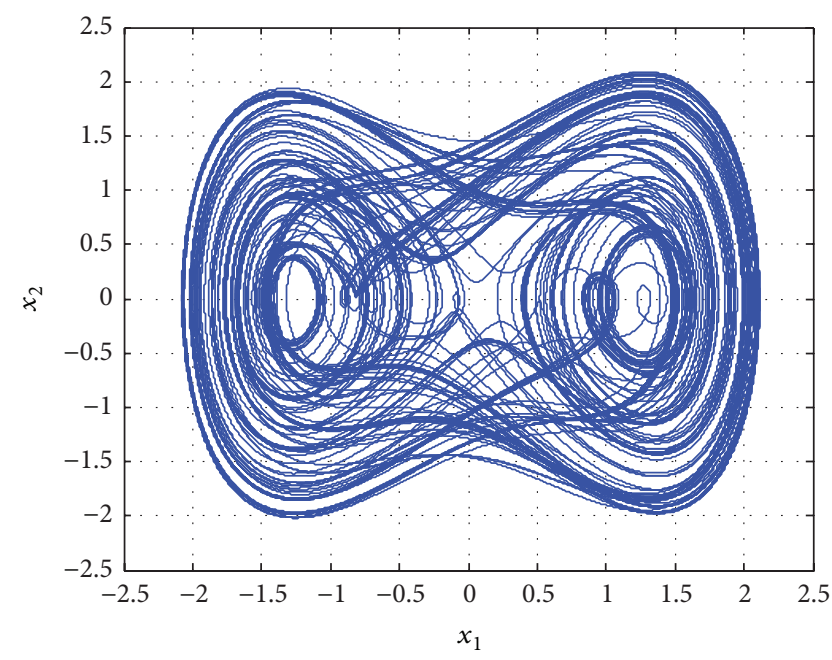

FIGURE 9: Duffing attractor with sinusoidal signal and digital image (Chichen-Itzá).

known frequencies and with a signal message. The main result is that using a perturbing sinusoidal signal the encryption level could be increased since the message signal is hidden not only in the spread spectrum of the chaotic system but also by the frequencies of the disturbance. This fact is what we consider an increment on the encryption level and security on the transmission. We corroborate the encryption using two examples: transmitting and audio signal and an image; in both cases the recovery information is the same as the original message, except in the transition time. In practical terms this algorithm could be implemented in real time electronic circuit and also in a DSP or a fast processor since the number of equations to be solved is small, depending on the chaotic system and the number of sinusoidal signals used. Finally, an extension of this work is the encryption with sinusoidal signals with unknown frequencies, in addition to the problem of encrypting and transmitting multiple messages.
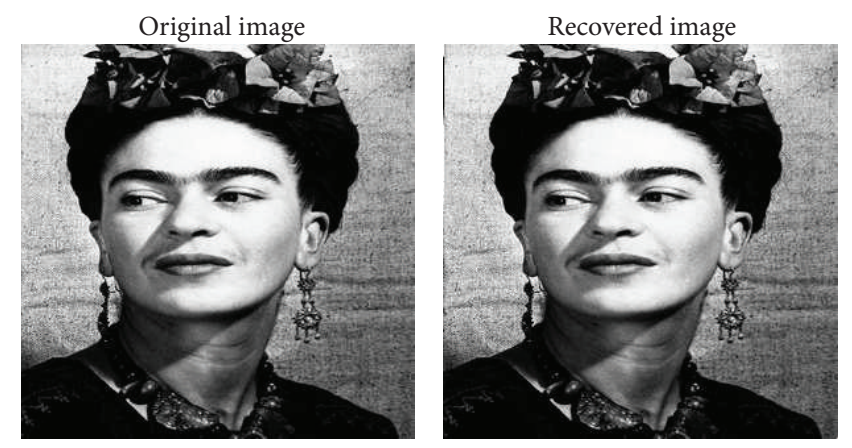

(a)
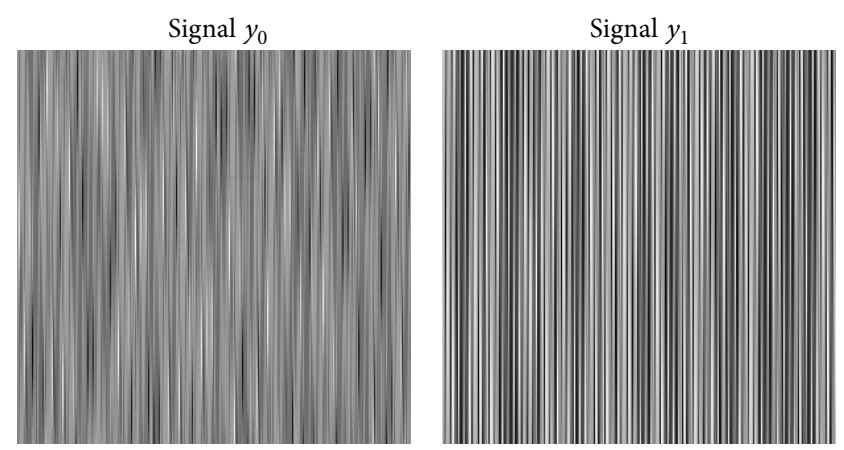

(b)

FIgURE 10: (a) Comparison between the original information and the recovered image. (b) Chaotic signals $y_{0}$ and $y_{1}$.
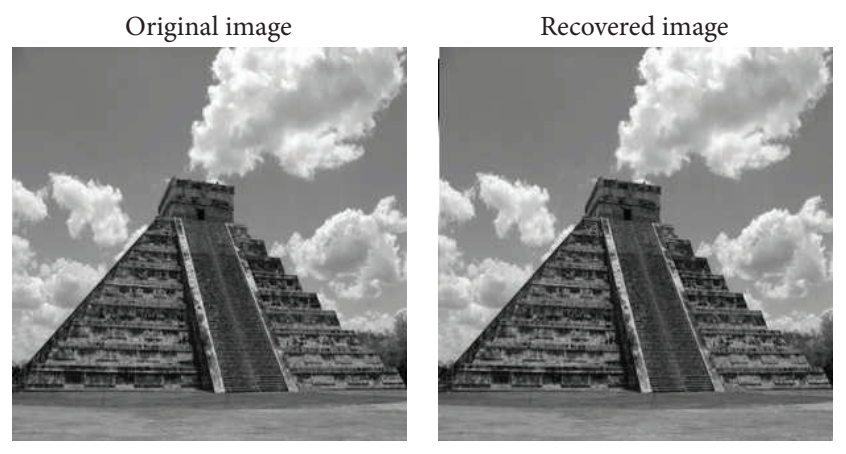

(a)
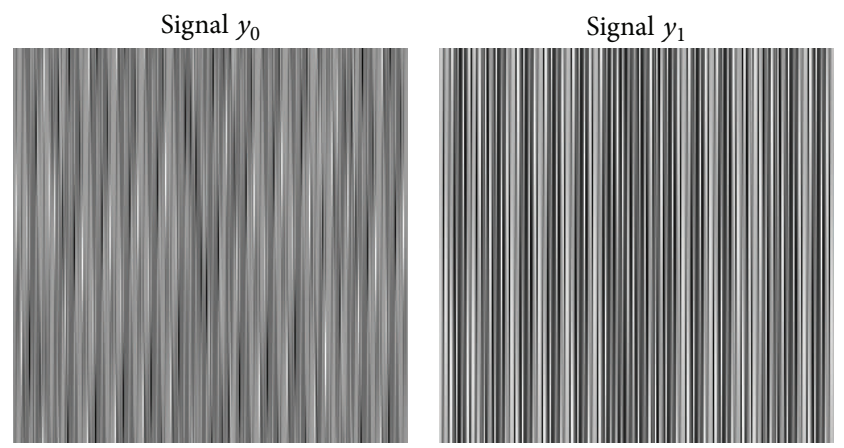

(b)

FIGURE 11: (a) Comparison between the original information and the recovered image. (b) Chaotic signals $y_{0}$ and $y_{1}$. 


\section{Conflict of Interests}

The authors declare that there is no conflict of interests regarding the publication of this paper.

\section{References}

[1] L. M. Pecora and T. L. Carroll, "Synchronization in chaotic systems," Physical Review Letters, vol. 64, no. 8, pp. 821-824, 1990.

[2] G. Alvarez and S. Li, "Some basic cryptographic requirements for chaos-based cryptosystems," International Journal of Bifurcation and Chaos in Applied Sciences and Engineering, vol. 16, no. 8, pp. 2129-2151, 2006.

[3] G. L. Gámez, "Synchronization of multi-scroll chaos generators: application to private communication," Revista Mexicana de Física, vol. 54, no. 4, pp. 299-305, 2008.

[4] L. Gámez-Guzmána, C. Cruz-Hernándeza, R. M. LópezGutiérrezb, and E. E. García-Guerrero, "Synchronization of Chua's circuits with multi-scroll application to communication," Communications in Nonlinear Science and Numerical Simulation, vol. 14, no. 9, pp. 2765-2775, 2009.

[5] K. M. Cuomo, A. V. Oppenheim, and S. H. Isabelle, "Spread spectrum modulation, and signal masking using synchronized chaotic systems," Tech. Rep., MIT Research Laboratory of Electronics, 1992.

[6] S. Li, G. Alvarez, Z. Li, and W. A. Halang, "Analog chaos-based secure communications and cryptanalysis: a brief survey," 2007, http://arxiv.org/PS_cache/arxiv/pdf/0710/0710.5455vl.pdf.

[7] F. Chiaraluce, L. Ciccarelli, E. Gambi et al., "A new chaotic algorithm for video encryption," IEEE Consumer Electronics Society, vol. 48, no. 4, pp. 838-843, 2002.

[8] G. R. Chen, Y. B. Mao, and C. K. Chui, "A symmetric image encryption scheme based on 3D chaotic cat maps," Chaos, Solitons and Fractals, vol. 21, no. 3, pp. 749-761, 2004.

[9] H. R. Karimi, "A sliding mode approach to $H_{\infty}$ synchronization of master-slave time-delay systems with Markovian jumping parameters and nonlinear uncertainties," Journal of the Franklin Institute. Engineering and Applied Mathematics, vol. 349, no. 4, pp. 1480-1496, 2012.

[10] B. Wang, P. Shi, H. R. Karimi, Y. Song, and J. Wang, "Robust $H_{\infty}$ synchronization of a hyper-chaotic system with disturbance input," Nonlinear Analysis. Real World Applications, vol. 14, no. 3, pp. 1487-1495, 2013.

[11] B. Wang, P. Shi, H. R. Karimi, and J. Wang, " $H_{\infty}$ robust controller design for synchronization of master-slave chaotic systems with disturbance input," Modeling Identification and Control, vol. 33, no. 1, pp. 27-34, 2012.

[12] A. Isidori and C. I. Byrnes, "Output regulation of nonlinear systems," IEEE Transactions on Automatic Control, vol. 35, no. 2, pp. 131-140, 1990.

[13] H. Richter, "Controlling the Lorenz system: combining global and local schemes," Chaos, Solitons and Fractals, vol. 12, no. 13, pp. 2375-2380, 2001.

[14] E. N. Lorenz, "Deterministic nonperiodic flow," Journal of the Atmospheric Sciences, vol. 20, no. 2, pp. 130-141, 1963.

[15] L. Charles Phillips and D. Royce Harbor, Feedback Control Systems, Prentice Hall, 4th edition, 2000. 


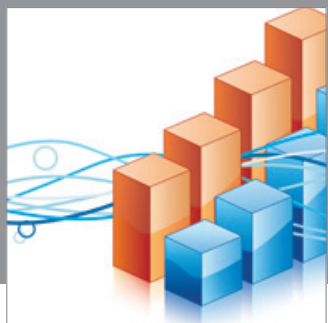

Advances in

Operations Research

mansans

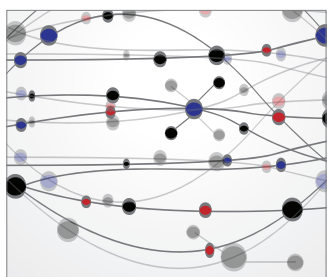

The Scientific World Journal
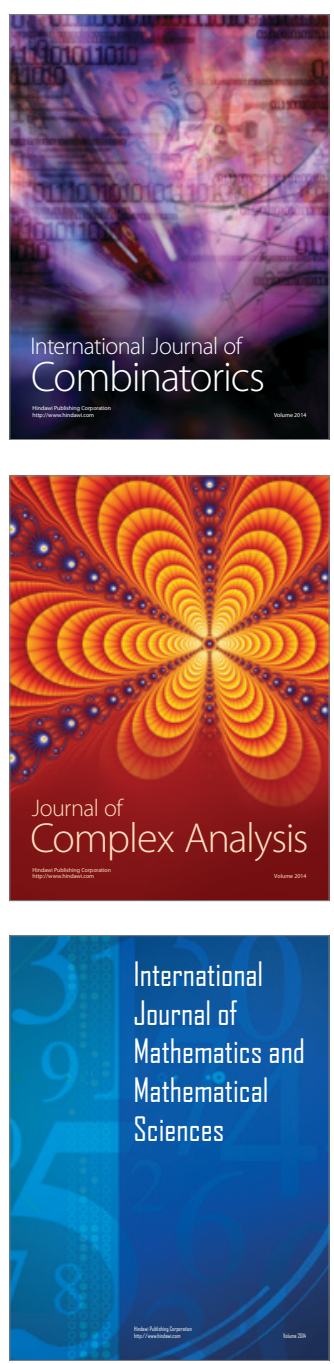
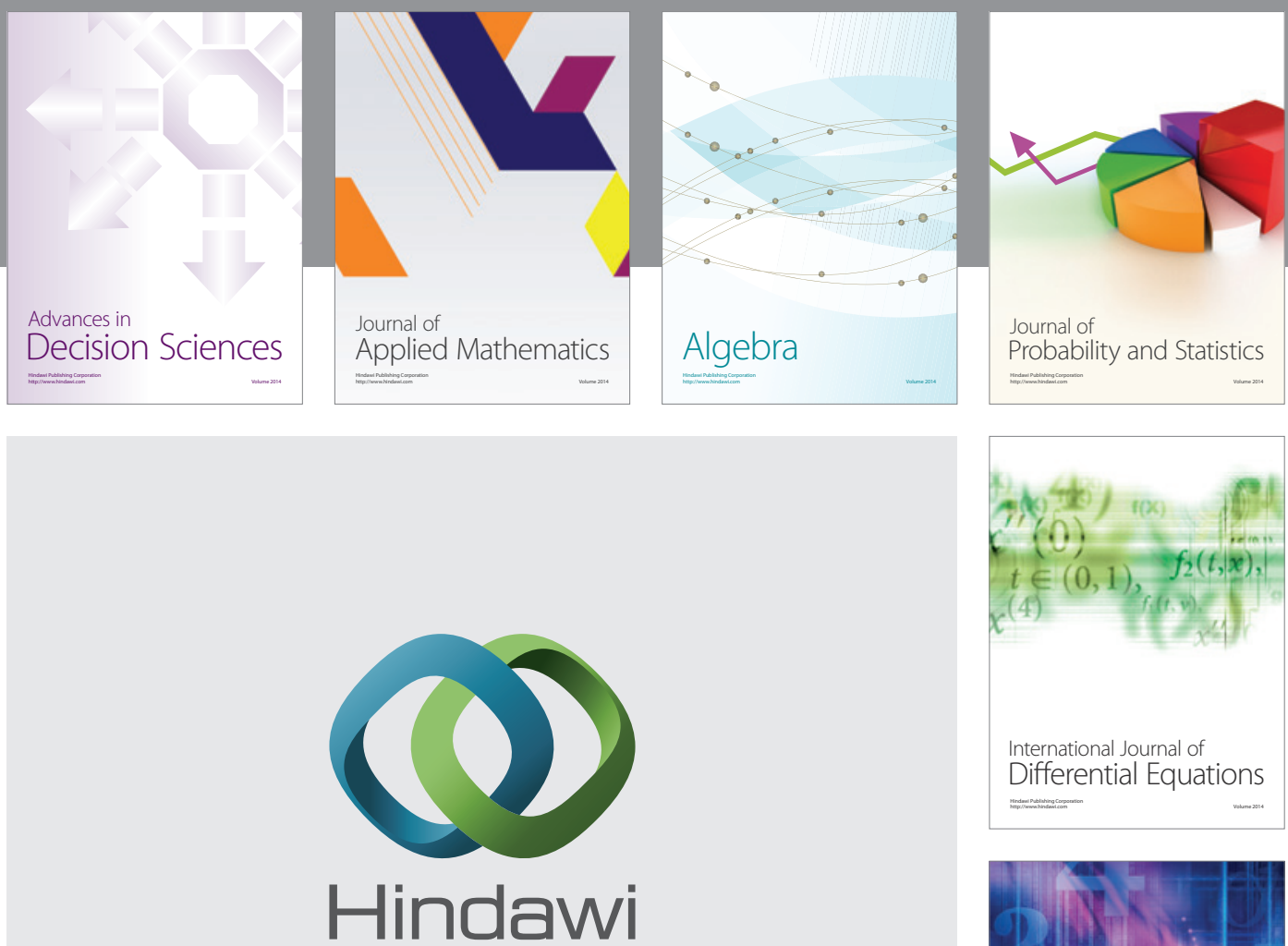

Submit your manuscripts at http://www.hindawi.com
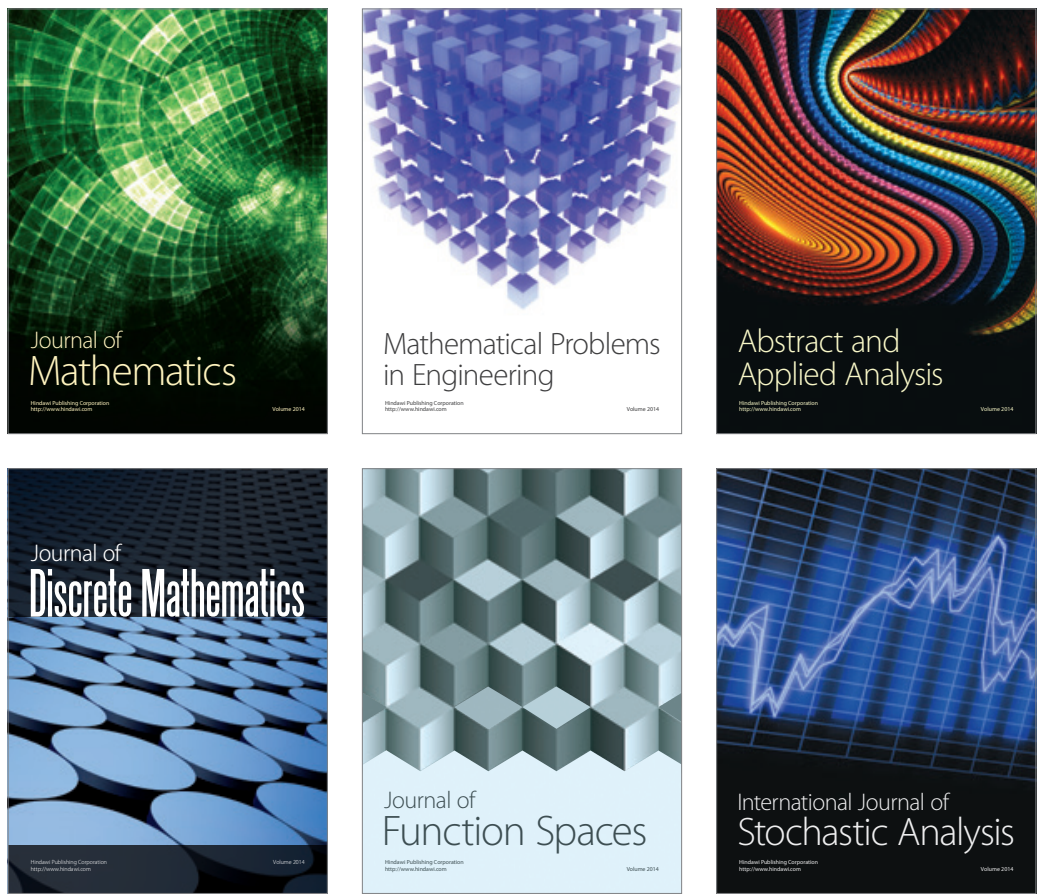

Journal of

Function Spaces

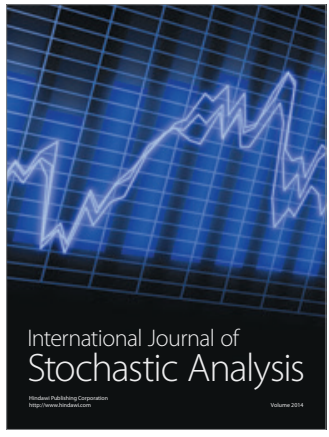

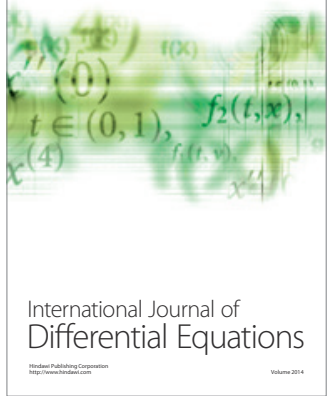
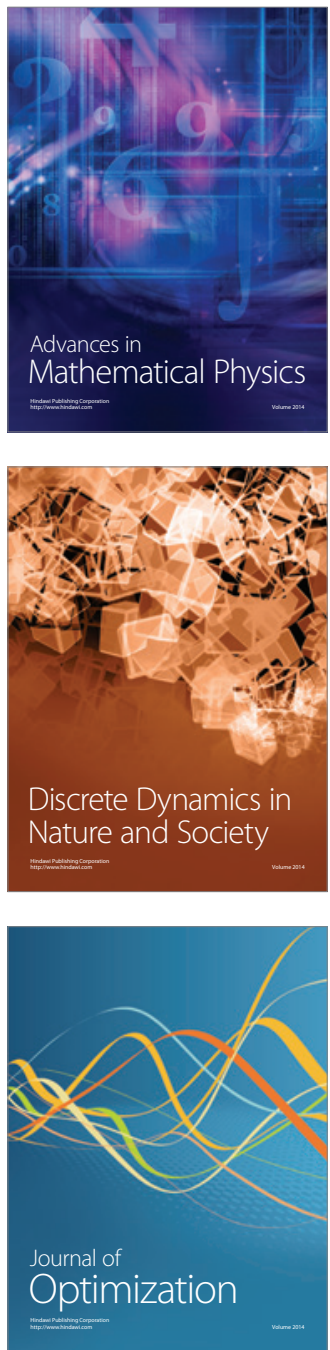